\title{
Gestion des ruptures d'approvisionnement de médicaments : perspectives en France, aux États-Unis et au Canada
}

\author{
Géraldine Ottino, Denis Lebel, Jean-François Bussières et Olivier Bourdon
}

\section{INTRODUCTION}

L es premiers écrits sur les ruptures d'approvisionnement de $u_{\text {médicaments datent des années soixante-dix }}^{1,2}$. Si les ruptures d'approvisionnement de médicaments ont fait partie de la réalité de la pratique pharmaceutique depuis plusieurs décennies ${ }^{3,4}$, elles sont devenues une préoccupation quotidienne des pharmaciens dans les années deux mille et ont dépassé les frontières de la littérature pharmaceutique ${ }^{5-9}$. Des sociétés savantes américaines et canadiennes ont notamment produit des lignes directrices afin que les professionnels de la santé encadrent le suivi de ces ruptures d'approvisionnement de médicaments ${ }^{10-12}$.

TIl existe de nombreuses causes de ruptures d'approvisionnement de médicaments, et la plupart de ces causes touchent plus d'une partie prenante, notamment les fabricants de matières premières, les fabricants de médicaments, les grossistes, les groupes d'achats, les prescripteurs, les pharmaciens hospitaliers et communautaires et surtout les patients. Une diffusion complète, accessible à tous et en temps réel, des données relatives aux ruptures d'approvisionnement (c.-à-d. état de tous les produits en rupture de stock et retirés du marché) peut contribuer à une gestion plus sécuritaire de ces événements pouvant être à l'origine d'erreurs médicamenteuses. La connaissance d'un risque de rupture d'approvisionnement permet d'informer les prescripteurs et ainsi d'avoir des prescriptions de sortie ou en ambulatoire comportant une solution de remplacement prévisible, permettant un gain de temps et une diminution de l'angoisse des patients.

Dans cette perspective, nous décrivons les obligations juridiques et les principaux sites web publics disponibles en France, aux États-Unis et au Canada pour la diffusion de l'état des ruptures d'approvisionnement de médicaments (tableau 1). Nous nous sommes limités à trois pays afin d'avoir une comparaison entre le Canada et deux autres systèmes réglementaires en Amérique et en Europe.

\section{MÉTHODE}

Il s'agit d'une étude descriptive transversale basée sur une revue documentaire effectuée en avril 2011 à partir du web, à l'aide de Google et de Google Scholar et des termes suivants : ruptures d'approvisionnement, ruptures de stock, médicaments en rupture, drug shortages, shortage of drugs, drug back orders. Dans notre démarche, nous étions à la recherche des obligations juridiques au sein de chaque pays ciblé et des sites web permettant d'accéder librement à la description de l'état des ruptures d'approvisionnement de médicaments (c.-à-d. sans nom d'usager et mot de passe). De plus, nous avons consulté systématiquement les sites web des autorités réglementaires de chaque pays (c.-à-d. Food and Drug Administration (FDA), Agence française de sécurité sanitaire des produits de santé (AFSSAPS) et Santé Canada). Nous avons en outre discuté avec des collègues pharmaciens au sein de chaque pays afin de nous assurer d'avoir un portrait complet de la situation.

\section{RÉSULTATS \\ France}

Compte tenu de l'augmentation soutenue du nombre de ruptures d'approvisionnement, l'AFSSAPS a mis en place en 2002 une procédure de notification des ruptures d'approvisionnement. En 2004, une disposition législative est adoptée, obligeant les fabricants de médicaments à déclarer à l'AFSSAPS les difficultés d'approvisionnement qu'ils rencontrent ou risquent de rencontrer, notamment pour des médicaments indispensables. La démarche proposée comporte cinq étapes, soit 1) le fabricant informe l'AFSSAPS de tout risque de rupture ou de toute décision d'arrêt de commercialisation sur un formulaire type ${ }^{14}$; 2) l'AFSSAPS examine le caractère indispensable du médicament; 3) en concertation avec l'AFSSAPS, le fabricant diffuse sur le site de l'Agence l'information sur la pénurie à l'intention des professionnels de la santé; 4) une recherche de solutions palliatives est réalisée 


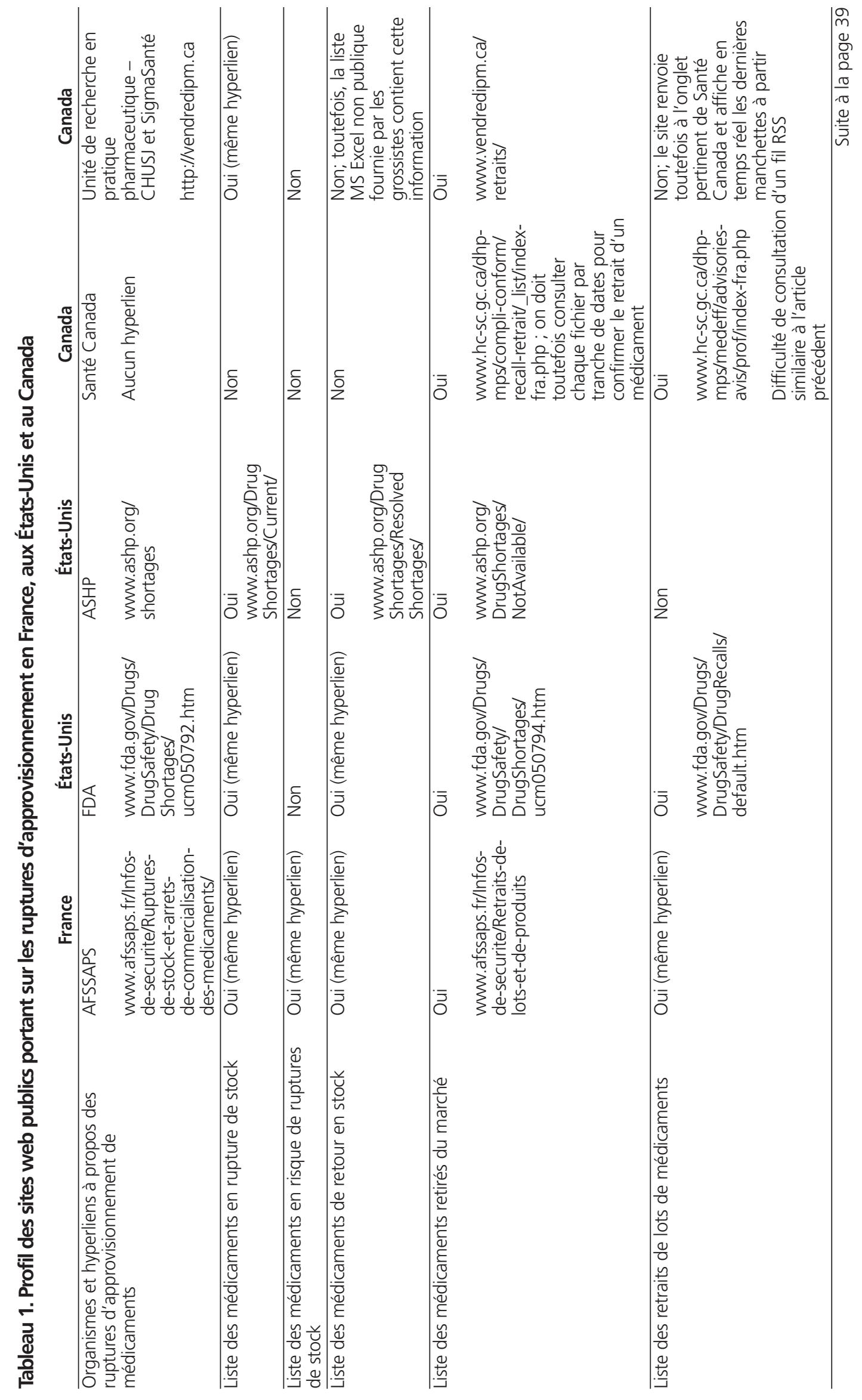




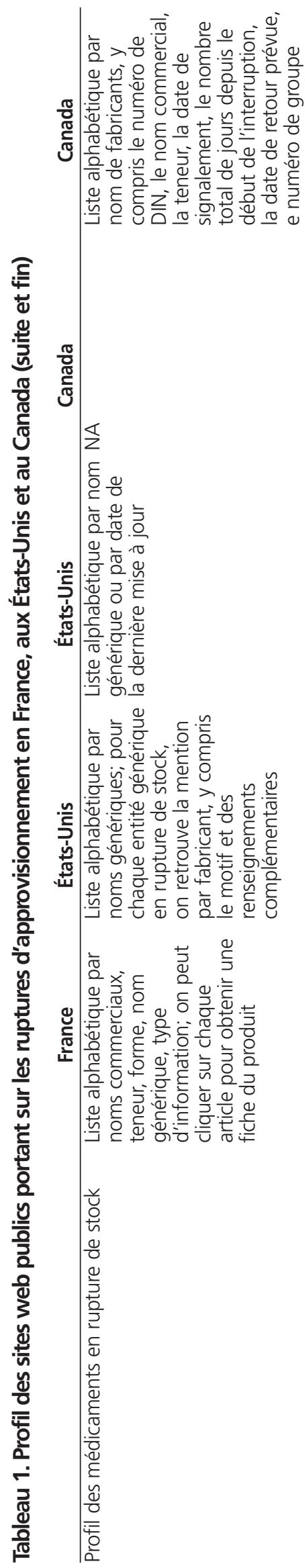

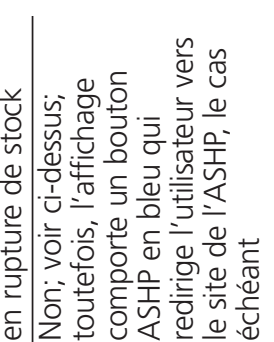

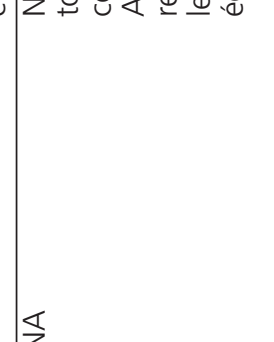

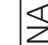

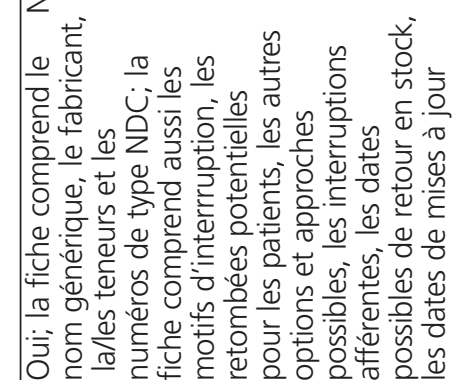

$\frac{\complement}{2}$

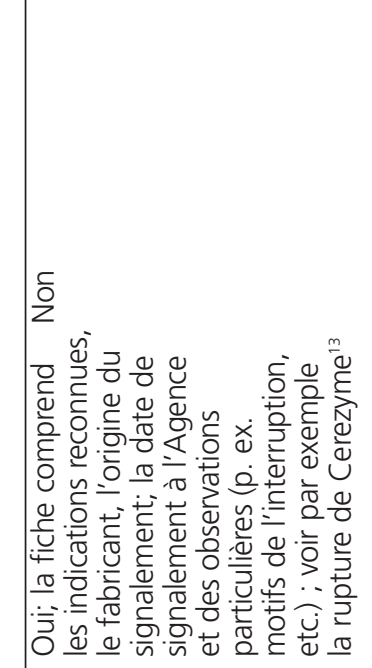

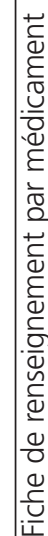

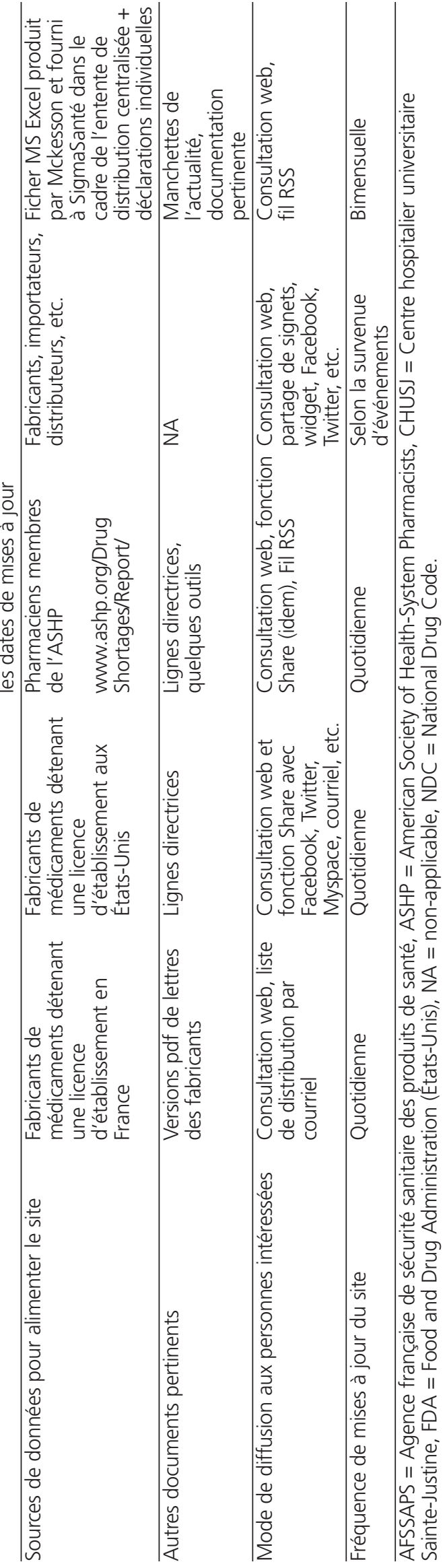


(p. ex. contingentement des stocks résiduels, mise à disposition de lots de marché étrangers ou de médicaments similaires, solutions thérapeutiques de remplacement); et 5) diffusion de l'information lors du retour en stock.

De plus, depuis mars 2007, les fabricants de médicaments ont l'obligation d'informer l'AFSSAPS au moins six mois avant la date envisagée d'un arrêt de commercialisation d'un médicament ne détenant pas d'autre possibilité thérapeutique. Ce changement législatif impose aussi le maintien du médicament sur le marché jusqu'à ce qu'une autre option soit disponible.

Sur le site web de l'AFSSAPS ${ }^{14}$, un onglet médicament et un sous-onglet portant sur les ruptures de stock de médicaments sont disponibles. Toutefois, ce sous-onglet ne concerne que les médicaments à usage humain, sans autre option thérapeutique disponible pour certains patients, ou dont les difficultés d'approvisionnement à l'officine ou à l'hôpital peuvent entraîner un risque de santé publique. Ces informations sont destinées aux professionnels de santé et aux patients. L'AFSSAPS précise que le laboratoire responsable de sa commercialisation en France est l'interlocuteur privilégié des personnes souhaitant des informations complémentaires. À noter que le site de l'Agence européenne du médicament (European Medicine Agency) ne comporte que la liste des médicaments approuvés, refusés ou retirés du marché15. En outre, le site permet le dépôt de versions électroniques (p. ex. fichiers pdf) des lettres du fabricant à un seul endroit, ce qui améliore considérablement la gestion documentaire et peut éliminer les multiples envois à de nombreux professionnels de la santé. À noter que le site ne comporte aucune statistique descriptive de l'état de situation.

\section{États-Unis}

La FDA reconnait l'importance de la problématique des ruptures d'approvisionnement de médicaments depuis plus d'une décennie ${ }^{16}$. Le Center for Drug Evaluation and Research a d'ailleurs révisé en 2006 son manuel de politiques et procédures relatif à la gestion des ruptures d'approvisionnement de médicaments ${ }^{17}$. Le manuel précise notamment les modalités de détermination de nécessité médicale (c.-à-d. identification des produits critiques dont la rupture peut avoir davantage de conséquences sur l'état de santé des patients) à l'intention du personnel de l'Agence afin, notamment, de justifier le recours à des médicaments provenant de pays étrangers. À la différence du contexte français, la notification de ruptures d'approvisionnement de médicaments par les fabricants de médicaments américains est un processus volontaire ${ }^{18}$. Le fabricant n'est pas tenu de déclarer les ruptures ni les risques de ruptures d'approvisionnement, les motifs ni les échéanciers.

Sur le site web de la FDA, un onglet précise les ruptures d'approvisionnement actives, les ruptures résolues, les retraits de marché et les procédures de déclaration volontaire. Dans sa foire aux questions, la FDA propose des statistiques descriptives de l'état de la situation ${ }^{19}$. Dans un bulletin de l'Institute for Safe Medication Practices publié en septembre 2010, on rapporte les résultats d'un sondage réalisé auprès de 1800 professionnels de la santé à l'été $2010^{20}$. Les sondés déclarent avoir peu ou n'avoir pas de renseignements en ce qui concerne la durée (85\%) et la cause $(83 \%)$ de la rupture d'approvisionnement ni les options disponibles $(70 \%)$. Ils notent des conséquences financières importantes $(78 \%)$, le recours à des ressources substantielles pour investiguer et résoudre la rupture d'approvisionnement pour les patients $(82 \%)$, l'identification de risques d'événements indésirables pour les patients (64\%).

À noter un projet de loi en discussion au sénat américain, qui vise à encadrer la détection et la gestion des ruptures de médicaments jugés critiques ${ }^{21}$.

En complément à la démarche de la FDA, l'American Society of Health-System Pharmacists (ASHP) a mis en place un volet de son portail consacré aux ruptures d'approvisionnement. Comme pour le site de la $\mathrm{FDA}^{16}$, les déclarations sont volontaires et proviennent cette fois des membres. Les deux organismes américains collaborent en partageant leurs données et leur expertise sur le sujet.

\section{Canada}

Au Canada, il n'existe aucune réglementation relative aux ruptures d'approvisionnement de médicaments. Ainsi, l'autorité réglementaire en place (Santé Canada) ne publie aucune politique ni ne diffuse d'information relative à la prévention, au suivi et à la gestion des ruptures d'approvisionnement de médicaments. Toutefois, d'autres paliers décisionnels peuvent introduire des obligations relatives à la prévention et à la gestion de ces ruptures. Par exemple, des groupes d'achats ont recours à des clauses de maintien de la responsabilité contractuelle en cas de ruptures d'approvisionnement assorties d'une obligation pour l'attributaire d'assumer la différence de coût pour l'achat d'une autre option durant la période d'interruption. Certains tiers payeurs publics peuvent envisager le retrait temporaire ou définitif d'une liste de remboursement en présence de ruptures d'approvisionnement soutenues et mal justifiées.

La problématique n'est pourtant pas nouvelle, l'Association des pharmaciens du Canada s'étant dotée d'un guide pratique sur la gestion des ruptures de médicaments dès $2001^{12}$. Cette association a d'ailleurs mis à jour ses lignes directrices en 2010 et mené une enquête auprès des pharmaciens de partout au pays. Un total de 427 pharmaciens ont répondu à l'enquête en octobre 2010, dont une proportion élevée de pharmaciens hospitaliers (environ $30 \%$ ) $^{22}$. L'enquête révèle notamment que plus de $80 \%$ des personnes interrogées ont eu, au cours de leur dernière période de travail, de la difficulté à obtenir au moins un médicament pour une ordonnance active (médiane de trois ordonnances) et près de $94 \%$ au cours de la dernière semaine (médiane de dix ordonnances). Pour chaque interruption vécue, les pharmaciens perdent en moyenne 30 minutes à trouver une solution. Moins de $15 \%$ des sondés estiment être avisés à l'avance de la pénurie, ce qui permet de planifier leur travail et des solutions de remplacement. Près de $90 \%$ des 
sondés estiment que le problème s'aggrave. Enfin, près de $70 \%$ d'entre eux estiment que les ruptures d'approvisionnement ont des conséquences fâcheuses sur la santé des patients.

Plusieurs fabricants font parvenir à leur clientèle (c,-à-d. grossistes, pharmaciens propriétaires, groupes d'achats, pharmaciens hospitaliers, etc.) une liste de médicaments en rupture de fourniture. Le problème est que ces envois sont erratiques, non uniformes (c,-à-d. forme et contenu très variables) et qu'ils doivent être consultés individuellement pour l'obtention d'un portrait global de la problématique. Certains groupes d'achat offrent une information similaire (p. ex. Medbuy envoie un courriel à ses membres, donnant accès, par son portail, à des chiffriers électroniques concernant chaque fabricant). Certains grossistes relaient aussi l'information (p. ex. McKesson publie sur son site transactionnel une mention de la rupture d'approvisionnement lors d'une commande et publie aussi une liste en chiffrier électronique agrégée bimensuelle des ruptures d'approvisionnement de médicaments et des retours en stock). Toutes ces approches ne sont pas optimales, et l'information publiée n'est pas forcément complète (p. ex. certaines listes ne comportent pas la date de mise à jour de l'information, la date prévue de retour en stock, la personne à contacter pour confirmer la donnée). Un seul site canadien offre une information publique complète (c.-à-d. tous les fabricants et toutes les ruptures de livraison connues) à toutes les parties prenantes (c.-à-d. professionnels de la santé et patients), soit http:// vendredipm.ca sans nom d'usager et de mot de passe (Figure 1). Le site comporte l'ensemble des médicaments en rupture d'approvisionnement, le nom du fabricant, la date de début de l'interruption, la date présumée de sa résolution lorsqu'elle est connue, le nombre total de jours de discontinuité depuis le début de l'interruption, le nom du médicament, sa teneur, sa forme, son format, le numéro de grossiste chez McKesson, le numéro d'ingrédient actif selon la banque de données des

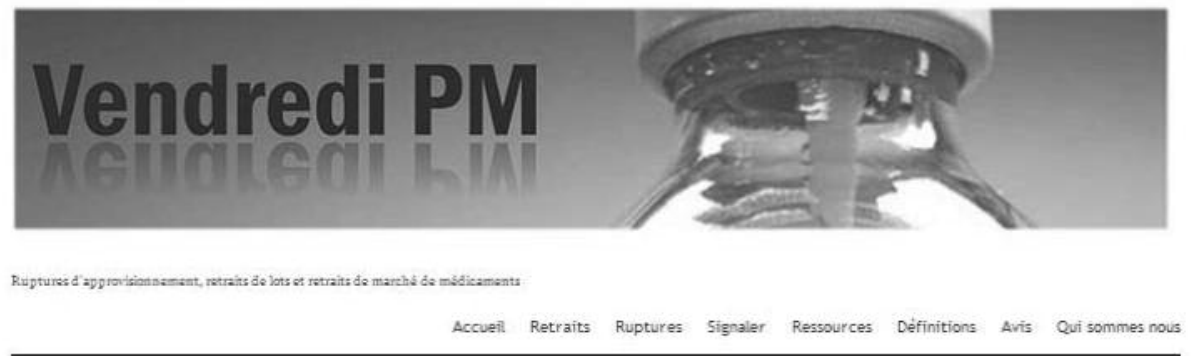

Ruptures d'approvisionnement en médicaments au Canada

OOBMENTS OFF

by asmin

Dans le cadre des activités du Comité des pharmaciens de Sigma Santé, nous publions

périodiquement les données relatives aux ruptures d'approvisionnement, aux ratraits de lots at

aux retraits de marché des médicaments au canada. sous iavitons les usagers a consulter lavis de mise en garde avant dutiliser les donnees publiées. Ce site comporte les sections suivantes:

+ Accueil

* Avis

* Définitions

\& Qui sommes nous?

* Rearources

Ruptures

Catte initiative est réalisee par 1Unité de recherche en pratique pharmaceutique, CMU SainteJustine avec la contritution đe Géraldine Ottino, Denis Lebel at Jean-François sussières.

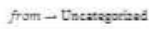

Ruptures en date du 5 octobre 2011

LEAYEA Cosmoms

Dond

by Denia

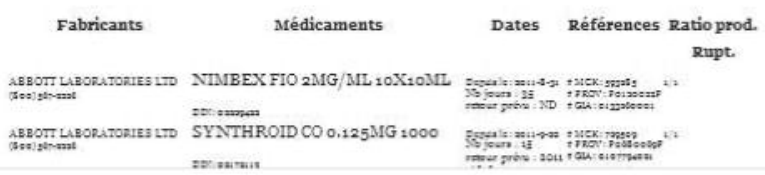

RESSOURCES EXTERNES

ASHP Drug Shortages

LAmerican Society of Health System Pharmacists propose une section détaillée de son site web sur les ruptures

d'approvisionnement de médicaments

FDA Current drug shortages

La Food and Drug Administration (FDA) comporte une section de son site WEB dédiée aux ruptures d'approvisionnement

Santé-Canada : Avis, mises en garde et retraits

Page principale des avis, mise en garde et retraits sur le site de Sante-Canada

DAVIS ET RETRAITS (SANTE CANADA)

Santé Canada evalue le risque cardiaque de citalopram à dose elevée

13 October 2011

Plavix (anticoagulant) : Nouvelles

reoommandations sur l'utilisation aveo des

IPP (antiacides)

22 September 2011

Trasylol (aprotinine) - Nouveaux

renseignements importants en matière

dinnocuite

21 September 2011

Agonistes de la GnRH : risque cardiaque chez les hommes traites pour le cancer de la

Figure 1. Extrait du site vendredipm.ca 
produits pharmaceutiques de Santé Canada, le ratio de produits similaires en rupture d'approvisionnement sur le marché canadien, le lien vers le site de l'ASHP lorsqu'une interruption similaire est en cours. Le site sera traduit en anglais sous peu.

\section{DISCUSSION}

Bien que la problématique des ruptures d'approvisionnement de médicaments ne soit pas nouvelle, les initiatives visant à faciliter leur prévention, leur gestion et leur résolution ne font que naître. Il est étonnant de constater qu'il n'existe pas de sites canadiens officiels d'un organisme réglementaire ou d'une association donnant libre accès en temps réel aux données relatives aux ruptures de disponibilité des médicaments.

Les dispositions adoptées en France et mises en place sur le site de l'AFSSAPS devraient inspirer les décideurs canadiens, parce qu'elles sont encadrées juridiquement par une obligation faite au fabricant de déclarer à l'avance les risques de manque de médicaments et parce qu'elles permettent un affichage public de l'information, incluant la correspondance entre le fabricant et sa clientèle. L'initiative québécoise de l'Unité de recherche en pratique pharmaceutique vise à optimiser la diffusion de l'information relative aux ruptures d'approvisionnement. Dans le retour en appel d'offre de SigmaSanté pour le contrat relatif aux médicaments, une clause sera ajoutée afin d'exiger la déclaration en ligne des ruptures d'approvisionnement par les fabricants participants. Cette disposition devrait permettre une mise à jour en temps réel de l'état de la situation par toutes les parties prenantes plutôt qu'une mise à jour bimensuelle de vendredipm.ca. En outre, des discussions avec l'Association des pharmaciens du Canada, la Société canadienne des pharmaciens d'hôpitaux et les deux principales associations représentant les fabricants de médicaments au Canada devraient permettre de concrétiser cette participation prévoyante des fabricants d'ici la fin de 2011. Espérons que ces initiatives contribueront à améliorer la gestion des ruptures d'approvisionnement de médicaments et à limiter les retombées négatives de ces interruptions sur la santé des patients.

\section{Références}

1. Stolar MH. Drug shortages. Science 1976;192(4235):94.

2. Kubica AJ, Bowman G. A statewide drug shortage reporting system [lettre] Am J Hosp Pharm 1976;33(7):628-631.

3. Myronuk LD, Meagher JB. Drug patent law reform and shortages. $C M A$ J 1993;149(9): 1225 .

4. Young D. Drug shortages frustrate health care providers. Am J Health Syst Pharm 2002;59(8):698-699, 703.

5. Inglis T. National drug shortages. Am J Nurs 2002;102(7):11.

6. Tyler LS, Fox ER, Caravati EM. The challenge of drug shortages for emergency medicine. Ann Emerg Med 2002;40(6):598-602.

7. Eggertson L. Continuing drug shortages affect North American patients. CMAJ 2010;182(18): E811-E812.

8. Steinbrook R. Drug shortages and public health. $N$ Engl J Med 2009; 361(16):1525-1527.

9. Traynor K. Drug shortages mount in 2010. Am J Health Syst Pharm 2010; 67(18):1492,1494.

10. American Society of Health-System Pharmacists, Council on Administrative Affairs. ASHP guidelines on managing drug product shortages. Am J Health Syst Pharm 2001;58(15):1445-1450.
11. ASHP Expert Panel on Drug Product Shortages; Fox ER, Birt A, James KB, Kokko H, Salverson S, Soflin DL, et al. ASHP guidelines on managing drug product shortages in hospitals and health systems. Am J Health Syst Pharm 2009;66(15):1399-1406.

12. Drug shortages. A guide for assessment and patient management. Ottawa (ON) : Association des pharmaciens du Canada; 2010. Publié au www.pharmacists.ca/cpha-ca/assets/File/cpha-on-the-issues/Drug ShortagesGuide.pdf. Consulté le 20 décembre 2011.

13. CEREZYME $400 \mathrm{U}$, poudre pour solution à diluer pour perfusionrisque de rupture de stock. Saint-Denis (France) : Agence française de sécuritaire sanitaire des produits de santé; 11 octobre 2011. Publié au www.afssaps.fr/Infos-de-securite/Ruptures-de-stock-et-arrets-decommercialisation-des-medicaments/CEREZYME-400-U-poudre-poursolution-a-diluer-pour-perfusion-Risque-de-rupture-de-stock. Consulté le 4 janvier 2012.

14. Formulaire de déclaration de rupture de stocks à l'intention des laboratoires. Saint Denis (France) : Agence française de sécuritaire sanitaire des produits de santé; 2008. Publié au www.afssaps.fr/Infos-desecurite/Ruptures-de-stock-et-arrets-de-commercialisation-desmedicaments. Consulté le 14 juin 2011.

15. European public assessment reports. London (UK) : European Medicines Agency. Publié au www.ema.europa.eu/ema/index.jsp?curl=/pages/ medicines/landing/epar_search.jsp\&murl=menus/medicines/medicines.jsp \&mid=WC0b01ac058001d124. Consulté le 14 juin 2011.

16. Drug shortages. Silver Spring (MD) : Food and Drug Administration. Publié au www.fda.gov/Drugs/DrugSafety/DrugShortages/default.htm. Consulté le 14 juin 2011.

17. Manual of policies and procedures: drug shortage management. Silver Spring (MD) : Drug and Food Administration, Center for Drug Evaluation and Research, Office of New Drugs; 2006. Publié au www.fda.gov/downloads/AboutFDA/CentersOffices/CDER/ManualofPoliciesProcedures/U CM079936.pdf. Consulté le14 juin 2011.

18. How to report a shortage or supply issue. Silver Spring (MD) : Food and Drug Administration; [mis à jour 2011]. Publié au www.fda.gov/ Drugs/DrugSafety/DrugShortages/ucm142398.htm. Consulté le 14 juin 2011.

19. Frequently asked questions about drug shortages. Silver Spring (MD) : Food and Drug Administration. Publié au www.fda.gov/Drugs/Drug Safety/DrugShortages/ucm050796.htm. Consulté le 14 juin 2011.

20. Drug shortages: national survey reveals high level of frustration, low level of safety. Med Saf Alerts 2010 septembre 23;15(19). Publié au www. ismp.org/Newsletters/acutecare/articles/20100923.asp. Consulté le 14 juin 2011.

21. Sen. Schumer touts bill to prevent drug shortages. The San Francisco Examiner;11 juin 2011. Publié au www.sfexaminer.com/news/health/2011/ 06/sen-schumer-touts-bill-prevent-drugshortages? category $=919968$ quicktabs_1=0. Consulté le 14 juin 2011.

22. Canadian drug shortages survey - final report. Ottawa $(\mathrm{ON})$ : Association des pharmaciens du Canada; 2010 décembre. Publié au www.pharmacists.ca/ content/About_CPHA/Whats_Happening/CPhA_in_the_News/CPhAD rugShortagesReport_Dec2010.pdf. Consulté le 14 juin 2011.

Géraldine Ottino est étudiante au programme D. Pharm. à I'Université Claude Bernard Lyon 1, et assistante de recherche à l'Unité de recherche en pratique pharmaceutique au CHU Sainte-Justine, Montréal, Québec.

Denis Lebel, B. Pharm., M. Sc., est adjoint aux soins pharmaceutiques, à l'enseignement et à la recherche à l'Unité de recherche en pratique pharmaceutique au CHU Sainte-Justine, Montréal, Québec.

Jean-François Bussières, B. Pharm., M.Sc., est chef du Département de pharmacie et de I'Unité de recherche en pratique pharmaceutique au CHU Sainte-Justine, et professeur titulaire de clinique à la Faculté de pharmacie de I'Université de Montréal, Montréal, Québec.

Olivier Bourdon, Pharm. D., Ph. D., APHP, est pharmacien à l'Hôpital Robert Debré, et professeur à I'Université Paris Descartes, Paris, France.

\section{Adresse de correspondance :}

Jean-François Bussières

Département de pharmacie

Centre hospitalier universitaire Sainte-Justine

3175, chemin de la Côte Sainte-Catherine

Montréal (Québec) H3T 1C5

Courriel : jf.bussieres@ssss.gouv.qc.ca 